\title{
An enhancer screen identifies a gene that encodes the yeast U1 snRNP A protein: implications for snRNP protein function in pre-mRNA splicing
}

\author{
Xiaoling C. Liao, ${ }^{1}$ Jie Tang, and Michael Rosbash, ${ }^{2}$ \\ Howard Hughes Medical Institute, Department of Biology, Brandeis University, Waltham, Massachusetts 02254 USA
}

In an enhancer screen for yeast mutants that may interact with U1 small nuclear RNA (snRNA), we identified a gene that encodes the apparent yeast homolog of the well-studied human U1A protein. Both in vitro and in vivo, the absence of the protein has a dramatic effect on the activity of U1 snRNP containing the mutant U1 snRNA used in the screen. Surprisingly, the U1A gene is inessential in a wild-type U1 RNA background, as growth rate and the splicing of endogenous pre-mRNA transcripts are normal in these strains that lack the U1A protein. Even in vitro, the absence of the protein has little effect on splicing. On the basis of these observations, we suggest that a principal role of the U1A protein is to help fold or maintain U1 RNA in an active configuration.

[Key Words: Yeast; U1 snRNP; pre-mRNA splicing]

Received November 4, 1992; revised version accepted December 28, 1992.

Pre-mRNA splicing is characterized by a pair of highly conserved transesterification reactions that remove intervening sequences from primary transcripts. In both yeast (Saccharomyces cerevisiae) and mammalian extracts, these reactions take place in a large complex called the spliceosome. Small nuclear ribonucleoprotein particles (snRNPs), as well as many protein factors, participate in the spliceosome assembly process and are required for pre-mRNA splicing. Each of the four splicing snRNPs (U1, U2, U4/U6, and U5) contains one or two small nuclear RNAs (snRNAs). In mammalian systems, where they are better characterized, each snRNP also contains 7-10 proteins. These include core snRNP proteins, common to all four snRNPs (e.g., Sm proteins), and snRNP-specific proteins (for reviews, see Maniatis and Reed 1987; Steitz et al. 1988; Green 1991; Guthrie 1991; Rosbash and Séraphin 1991; Ruby and Abelson 1991).

Accumulating evidence indicates that in mammals as well as in yeast, $\mathrm{U} 1$ is the first snRNP to interact with pre-mRNA and that this interaction is required for the subsequent binding of U2 snRNP and the U4/U6/U5 tri-snRNP (Legrain et al. 1988; Ruby and Abelson 1988; Bartkiewicz et al. 1989; Barabino et al. 1990; Lamm et al. 1991; Séraphin et al. 1991|. In yeast extracts this interaction has been shown to commit the pre-mRNA to the splicing pathway (Séraphin and Rosbash 1989), and re-

\footnotetext{
${ }^{1}$ Present address: Department of Microbiology and Immunology, University of California, San Francisco, California 94143.

${ }^{2}$ Corresponding author.
}

cent evidence indicates that a similar situation is likely to obtain in mammalian systems (Michaud and Reed 1991; Jamison et al. 1992). All of these results suggest that Ul snRNP plays a prominent role in the early interactions between the pre-mRNA substrate and the splicing machinery. Many proteins, both snRNP and non-snRNP factors, undoubtedly mediate these interactions.

Mutant screens for yeast splicing factors have enjoyed considerable success (Ruby and Abelson 1991), yet the one identified gene that might contribute to these early assembly-recognition events (encoding a putative U1 $70 \mathrm{~K}$ protein) was identified through random sequencing of the yeast genome (Smith and Barrell 1991). To ameliorate this deficiency, we have exploited a synthetic lethal or enhancer strategy to screen for mutants that would make a viable strain containing a mutant $U 1$ RNA die (thus, the name mud for mutant-u-die).

This general strategy has been exploited previously by other investigators (Huffaker et al. 1987; Kranz and Holm 1990; Simon et al. 1991; Frank et al. 1992) and was dictated here by several considerations. First, we were concerned that certain splicing functions might not be easily revealed in traditional mutant screens. Some factors might mutate only rarely to temperature sensitivity, and the function of others might be buffered by redundant activities. In addition, growth rate is an insensitive indicator of splicing activity (Pikielny and Rosbash 1985), and this is likely to be equally true for mutations targeted to U1 snRNP (Séraphin and Rosbash 1989). Sec- 
ond, the synthetic lethality was dependent on a mutant Ul snRNA, hopefully making it more focused on early splicing events and U1 snRNP function than more general screens for splicing defective mutants/Vijayraghavan et al. 1989|. The genetic screen was augmented by an in vitro, biochemical approach; extracts were prepared from candidate mutant strains and assayed for U1 snRNP function. Among the first mutants examined, one strain was dramatically defective in U1 snRNP complex formation. Cloning and sequencing of the mutant gene identified the apparent yeast homolog of the well-studied human Ul snRNP protein, UlA. Characterization of strains deleted for the U1A gene indicated that the gene is inessential. Even in vitro, splicing and Ul snRNP activity in the absence of the UlA protein were indistinguishable from what was observed in wild-type extracts. Taken together with the synthetic lethality, these observations suggest that the UlA protein and part of the U1 RNA have overlapping functions to help fold or maintain the universally conserved structural elements of U1 RNA in an active configuration.

\section{Results}

Because the initial strain subjected to mutagenesis contained a U1 RNA gene with a deletion of the yeast core region, we hoped that this strategy might also provide some insight into the function of this domain of the yeast U1 molecule. Although neither present in vertebrates nor essential for yeast, these structural elements are conserved among U1 snRNAs from different yeast species (Kretzner et al. 1990) and contribute to yeast U1 snRNP function (Liao et al. 1990). In addition to a deletion of the yeast core region, we included a point mutation in the conserved A loop in the strain subjected to mutagenesis so as not to restrict the mutant search to proteins that might interact exclusively with the yeast core region (Fig. 1A).

In the scheme for the genetic screening of mud mutants (Fig. 1B), the chromosomal Ul gene was deleted. The essential Ul function is provided by two plasmids: One contains the URA3 marker and the GAL-U1 gene, and the other contains the TRP1 marker and a mutant U1 gene carrying a deletion of the yeast core region ( $\triangle \mathrm{YC}$ ), combined with a point mutation of the universally conserved A loop (Fig. 1B; Liao et al. 1990). In the presence of glucose, which represses expression from the GAL-U1 gene, this merodiploid strain is strictly temperature sensitive, consistent with the previously described properties of the mutant U1 gene (Liao et al. 1990). Similarly, this strain grows at $30^{\circ} \mathrm{C}$ but not at $37^{\circ} \mathrm{C}$ when replicaplated to 5-fluoro-orotic acid (5-FOA) plates (to select for loss of the GAL-U1 plasmid) (Boeke et al. 1984).

The starting strains were mutagenized by UV or EMS and the viable colonies were replica-plated to selective medium (5-FOA). The mud mutants were defined by their inability to grow in the absence of the wild-type Ul-containing plasmid, even at the permissive temperature $\left(30^{\circ} \mathrm{C}\right)$. These strains were collected from the mas- ter plates and checked for their inability to grow on glucose. From 34,000 viable colonies, 178 putative mud mutants, comprising at least 16 complementation groups, were defined by this strategy.

\section{The mud1-1 mutant}

To assay for effects of the mud mutants on in vitro splicing, we made extracts from many of the putative mutant strains after glucose depletion of the wild-type U1 snRNA. Although incubation in glucose ultimately leads to cell death, overnight incubation, which allows $5-10$ rounds of cell division, dilutes the wild-type U1 snRNPs, and promotes the in vivo assembly and accumulation of Ul snRNPs that contain only the mutant U1 snRNA. The cells then contain an effectively homogeneous mutant Ul snRNP population whose function can be assayed in vitro after extract preparation (Séraphin and Rosbash 1989; Liao et al. 1992).

Although several mutations affected Ul snRNP activity qualitatively or quantitatively, one strain (mud1-1) showed a striking decrease in splicing (data not shown) and in U1 snRNP-pre-mRNA complex formation (Fig. 2A). In contrast, extracts derived from the control strain showed a single major band, characteristic of strains containing the mutant U1 $\Delta \mathrm{YC}$ (Liao et al. 1992). The lack of in vitro U1 snRNP activity was the result of the mud1-1 mutation because the phenotype was rescued by transformation with the MUD1 gene (Fig. 2A and see below). As the mutant U1 snRNA accumulated nearly normally during the overnight incubation in glucose in the mud1-1 strain (Fig. 2B), we interpret the mutation as affecting U1 snRNP activity rather than Ul snRNP assembly or accumulation. This interpretation is supported by the observation that the mutant Ul snRNA is still immunoprecipitated normally by antibodies directed against the $\mathrm{U} 170 \mathrm{~K}$ protein (data not shown).

To assay for in vivo splicing defects, we examined several intron-containing transcripts from mud1-1 strains after glucose depletion of the wild-type U1 snRNA population. Primer extension analysis of pre-U3A and preU3B RNAs (Myslinski et al. 1990; Zavanelli and Ares 1991) showed a dramatic increase in level that was mud1-1 specific (Fig. 3, lane 2), that is, glucose depletion in other mud mutant backgrounds (e.g., mud3-1) showed no comparable pre-mRNA increase (Fig. 3, lane 1). The accumulation of pre-U3 RNA, like all of the in vitro and in vivo phenotypes of the mud1-1 strain, was reversed by the cloned MUD1 gene (data not shown). This indicates that the splicing defects observed after depletion of the wild-type U1 snRNA were linked to the lethal phenotype of mud1-1; in all likelihood, the combination of the mud1-1 allele and the mutant U1 snRNA leads to in vivo splicing defects responsible for the lethality.

The mudl gene encodes the yeast U1 snRNP-specific A protein

The wild-type MUD1 gene was cloned by rescuing the growth defect of the original mud1-1 mutant strain (con- 

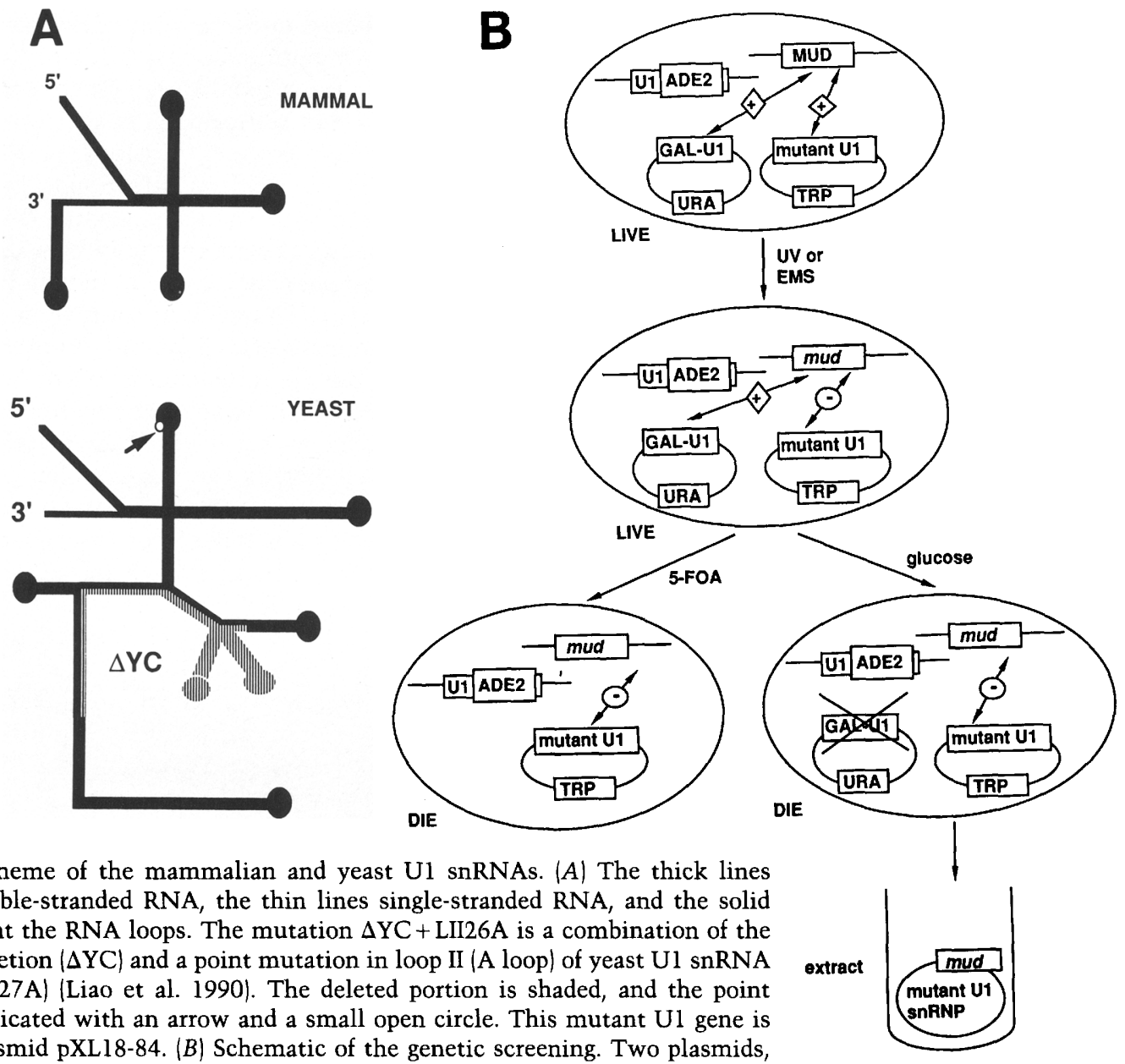

Figure 1. Scheme of the mammalian and yeast U1 snRNAs. $(A)$ The thick lines represent double-stranded RNA, the thin lines single-stranded RNA, and the solid ovals represent the RNA loops. The mutation $\triangle Y C+$ LII26A is a combination of the yeast core deletion $(\triangle \mathrm{YC})$ and a point mutation in loop II (A loop) of yeast Ul snRNA (formerly LII-27A) (Liao et al. 1990). The deleted portion is shaded, and the point mutant is indicated with an arrow and a small open circle. This mutant Ul gene is carried in plasmid pXL18-84. (B) Schematic of the genetic screening. Two plasmids, one carrying the wild-type U1 gene under the control of GAL-UAS (GAL-U1) and the other carrying the mutant U1 gene, are shown with their selectable markers. The interactions between the gene product of the MUD (or mud after mutagenesis) locus and the two types of Ul snRNPs are represented by the two-way arrows pointing to the corresponding loci. $(+\mid$ Interactions sufficient for cell growth (LIVE); $(-)$ a failure of interaction or aberrant interactions that result in cell lethality (DIE). Extracts can be made from the strain expressing the mud mutation after overnight growth in glucose-containing medium, and these extracts have a nearly homogeneous mutant U1 snRNP population (see text), the composition and activity of which is under the influence of the mud mutation.

taining the mutant U1 RNA) with a centromere-based library. Only two sets of plasmids were recovered: one contained overlapping sequences that encoded the wildtype U1 RNA gene, and the other contained different overlapping sequences that encoded the MUD1 gene. Subclones were generated, and a $1.5-\mathrm{kb}$ HindIII-HindIII fragment was able to rescue the mud1-1 mutation. In parallel, a linker insertion strategy was used to locate the relevant open reading frame (ORF) (Legrain et al. 1991). These linkers later served as primers to sequence doublestranded DNA in opposite directions. The entire 1.5-kb subclone of the MUD1 gene was sequenced in both directions. The internal region of the $1.5 \mathrm{~kb}$ subclone contained a single long ORF with a functional intron at its $5^{\prime}$ end (Fig. 4A).

The initiating methionine codon is located in the proper context for translation initiation (Donahue and Cigan 1990). Computer-aided searches of protein se- quence data bases identified the human U1A protein as the only close relative of Mud1. The relationship is highly significant, and both proteins contain two RNAbinding domains (RBDs) separated by spacer regions of similar lengths (115 amino acids for yeast vs. 120 amino acids for human). In addition, the monoclonal antibody 12CA5 specifically immunoprecipitated U1 RNA from extracts derived from strains that contain a hemagglutinin (HA) epitope-tagged version of the yeast UlA protein (Wilson et al. 1984; Abovich et al. 1990). The 12CA5 antibody recognizes the 9-amino acid epitope derived from the influenza virus HA protein. The results were comparable with those obtained with an HA epitopetagged version of the $\mathrm{U} 170 \mathrm{~K}$ protein (Fig. 4B).

The homology between the yeast and the human proteins begins within the $\beta 2$ region of the amino-terminal RBD and continues until the end of the protein (Fig. 4C). The second RBD is the most highly conserved portion of 
Liao et al.

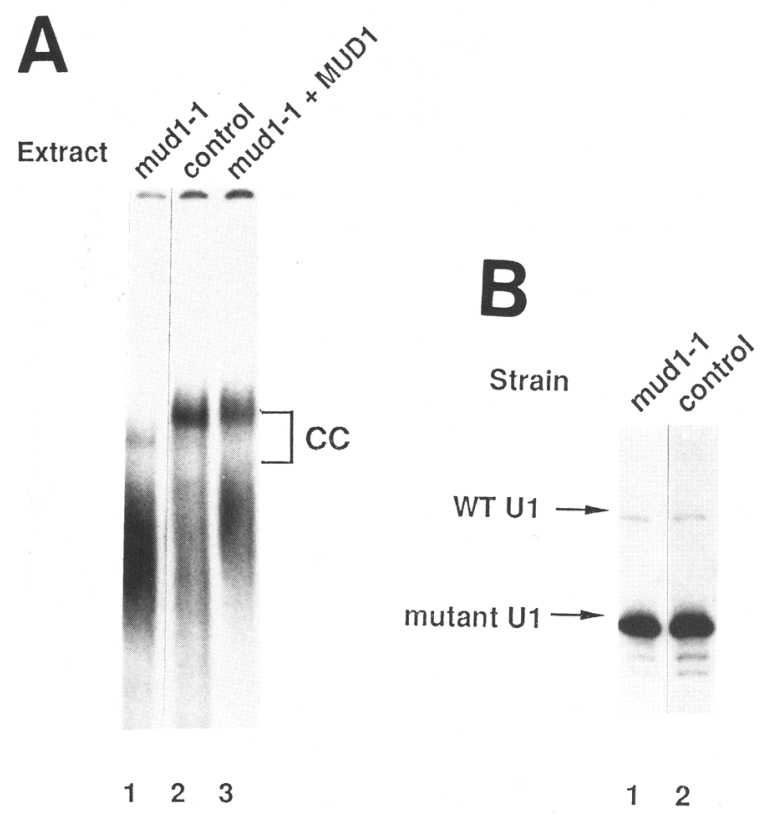

Figure 2. mud1-1 abolishes Ul snRNP activity in combination with the mutant U1 snRNA. (A) Extracts from strain Ym8 (XLY219 with the mud1-1 mutation) and the control strains (XLY219 or Ym8 plus the cloned MUD1 gene) were grown in glucose-containing medium for $16 \mathrm{hr}$ and assayed for commitment complex assembly after U2 killing (Liao et al. 1992). The position of the commitment complexes $(\mathrm{CC})$ is marked with a bracket. (+MUD1) The mud1-1 strain transformed with the wild-type MUD1 gene. $(B)$ The mutant U1 snRNA accumulates normally in the mud1-1 strain. The mutant and control strains were grown in glucose-containing medium for $16 \mathrm{hr}$ before RNAs were prepared and analyzed by primer extension. The positions of the wild-type Ul snRNA (WT U1) and the mutant Ul snRNA (mutant U1) are indicated by arrows.

the protein, and there is $36 \%$ identity and $64 \%$ similarity between the yeast and the human proteins in this region. The computer searches failed to identify the very similar U2 snRNP protein U2B". This is likely the result of the much different spacing between the two RBDs in U2B" (66 amino acids) and the three small regions of Mud1 that are conserved with human U1A but not with U2B" (identified in boldface type in Fig. 4C). There are no comparable regions that are more conserved with U2B" than with U1A. On the basis of this conservation and the biochemical assays, we propose that MUD1 encodes the yeast U1A protein.

The human U1A protein has been an object of intense study. Its amino-terminal RBD has been shown to bind to the B loop of human U1 snRNA, and the structure of this RBD has been established by X-ray crystallography (Nagai et al. 1990) and by nuclear magnetic resonance (NMR) spectroscopy (Hoffman et al. 1991). The loop 3 subregion of this RBD contributes to specificity and is substantially different in amino acid sequence between U1A and U2B" (Scherly et al. 1990); this region of the yeast protein is more similar to U1A than to U2B" (Fig $4 C_{;}$SRS at amino acids 68-70). There is also much more conservation between the amino-terminal RBDs of the two proteins when amino acids known or suspected to contact the human B loop RNA sequence are examined (Scherly et al. 1990, 1991; Jessen et al. 1991); of these 13 amino acids, 8 are identical in Mud1, and 2 more have conservative amino acid substitutions. This contrasts with an overall $24 \%$ sequence identity and $47 \%$ sequence similarity between the two RBDs. These considerations suggest that the as yet undefined B loop equivalent in yeast U1 RNA may bear some resemblance to its mammalian counterpart.

Yet an optimal alignment of the two proteins generated a substantial insertion in the loop 2 region of this RBD (Fig. 4C). As no well-defined stem-loop is apparent in the general region of the yeast molecule that corresponds to the location of the metazoan B loop and as the precise B loop sequence does not exist in the yeast UI

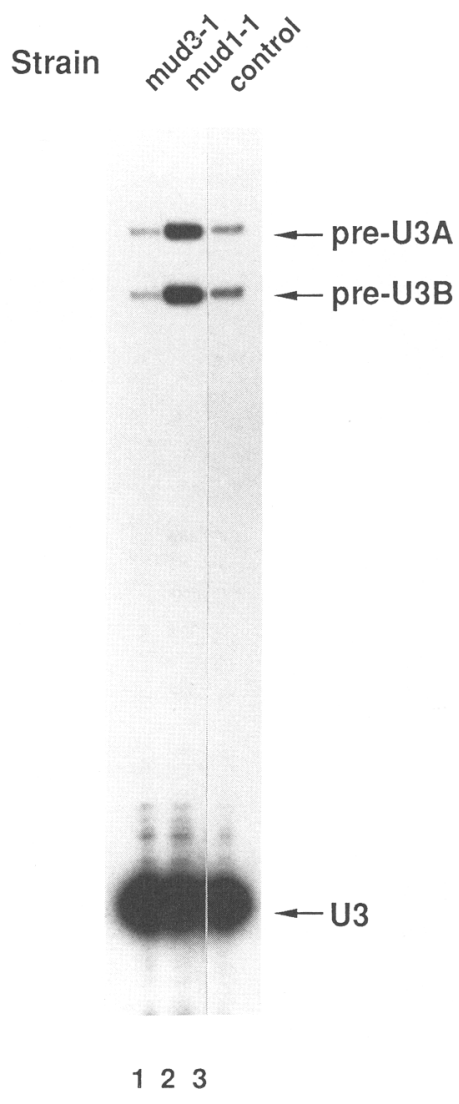

Figure 3. mud1-1 increases the U3 primary transcript levels in combination with the mutant U1 snRNA. The RNA samples from strains grown in glucose-containing medium for $16 \mathrm{hr}$ were analyzed by primer extension using oligonucleotide DT1967: 5'-CCAAGTTGGATTCAGTGGCTC-3'. The mutant strains were XLY219, with the mud1-1 mutation and XLY216 with a less well-characterized mud3 mutation as shown above the lanes. The control strain is XLY219. The positions of the precursor U3 RNA (pre-U3A and pre-U3B) and mature U3 snRNA (U3) are indicated with arrows. A lower exposure indicated that there was no detectable difference in the levels of mature U3 mRNA among the three lanes. 
A

AAGCTTTCAG TGAATACCCA CCATTAGGTA GATTCGCTGT CAGAGACATG AGACAAACTG TCGCTGTCGG TGTTATCAAG

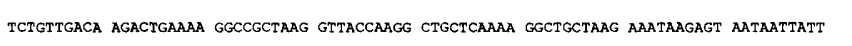

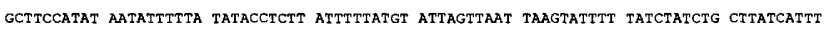
TCTTTTCATA TAGGGGGGT TGGTGTTTTC TTGCCCATCA GATTGATGTC CTCCAACTCG GCACTATTTT ACAAAGGGT TITTTGTAAG AGAAGGAGAA GACAGATACT AAACCATACG ITACTCGAAA CAAAAAAAAA AAAAATGGAA AAAGCTGCTA

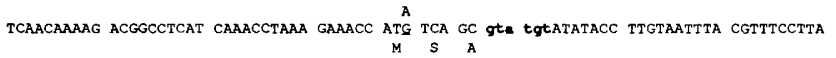

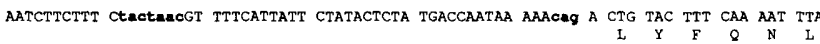
CCC AGT AGG CCA GCA AAT AAA GAA AAT TAT ACC AGA TTA CTT CTG AAA CAC ATT AAT CCC AAC AAC AaG TAT GCC ATT AAT CCG TCG CTA CCC CTT CCC CAC AAT AAA TTA CAA ATA TCA TCA CAA CCG CTG

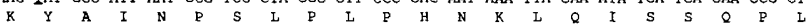
ATG TTA CTA GAT GAT CAA ATG GGC CTT CTT GAA GTT TCT ATT TCA AGA TCA TCA AAG ATG ACT AAC $T$ (mud1-1)

CAA GCA TTT TTA ACG TTT GTC ACT CAA GAA GAA GCA GAC CGG TTT CTA GAA AAA TAC ACG ACA ACA $\begin{array}{lllllllllllllllllllllll}I & T & F & V & T & Q & E & E & A & D & R & F & L & E & K & Y & T & T & T & \end{array}$

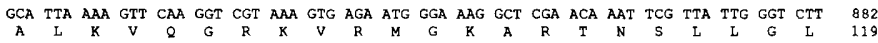
TCA ATA GAA ATG CAA AAA AAA AAA GGT AAT GAC GAA ACG TAC AAC CTT GAT ATA AAG AAG GTG CTT 948 $\begin{array}{lllllllllllllllllllllll}K & A & R & K & \text { L } & K & R & K & \text { L } & R & \text { S } & \text { D } & \text { D } & \text { I } & \text { C } & \text { A } & K & K & F & R & \text { L } & K & 163\end{array}$

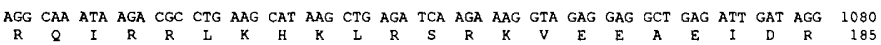

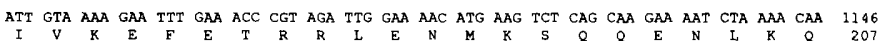
TCG CAG AAA CCT CTT AAG CGG GCT AAA GTG TCC AAT ACA ATG GAA AAT CCA CCG AAC AAA GTC CTT 1212

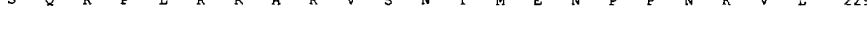

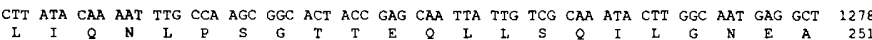
TTA GTT GAA ATC AGA TTA GTT AGC GTT CGT AAC CTA GCT TTC GTG GAA TAC GAG ACC GTT GCT GAT 1344

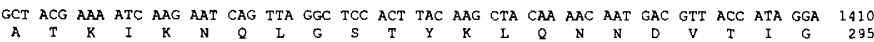
TTT GCT AAG TAG AATTTCCTTT GCGGAaGtat ACCTCGaGta AaGAAATTCA CAGATAAATt TGAATAACGT 1482 tctccattat gaAtaAatat gtattTacaA GctT

C yeast $1 \quad$ MS ALYFQN LPSRPAN KENYTRLLLKHI NPNNKYAINPSL 39

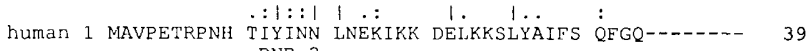
RNP - 2 $\beta 2$
40 PLPHNLQISSQRLMLLDDQMG LLEVI SRSSKMT NQAFLTF VT QE $\alpha 2 \quad$ loop5 $\quad \beta 4$ 86 EADRFLIEKY TTTALKVQGRK VRMGKA RTNS-----LLGLSIEMQKKKG 128

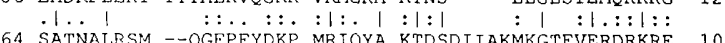

129 NDETYNLD - - IKKVLKARKLKRKLRSDDICAKKFRLKRQIRRLKHKLRSR 176

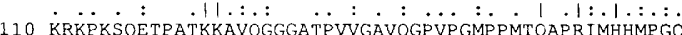

177 KVEEAEIDRI-VKEFETRRLENMKSQQENLKQSQKPLKRAKVSNTM ENPPN 226

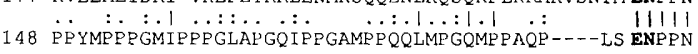
207

$\begin{array}{ccccccc}\beta 1 & \text { loop } & \alpha 1 & \text { loop2 } & \beta 2 & \text { loop3 } & \beta 3\end{array}$

227 KVLLIQN LPSGTT EQLLSQILGN-E ALVE IR-LVS V-R NLAFVEY 268

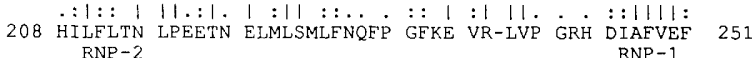
RNPRNP - 1

$\begin{array}{lll}\alpha 2 & \text { loop5 } \quad \beta 4\end{array}$

269 ET VADATKIKNQL GSTYKL-QNND VTIGFA K*

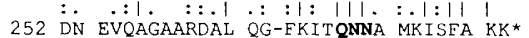

AAA GCA AGG AAA CTT AAA CGC AAG TTA CGT AGT GAT GAT ATA TGC GCC AAA AAG TTC AGG CTT AAA 1014

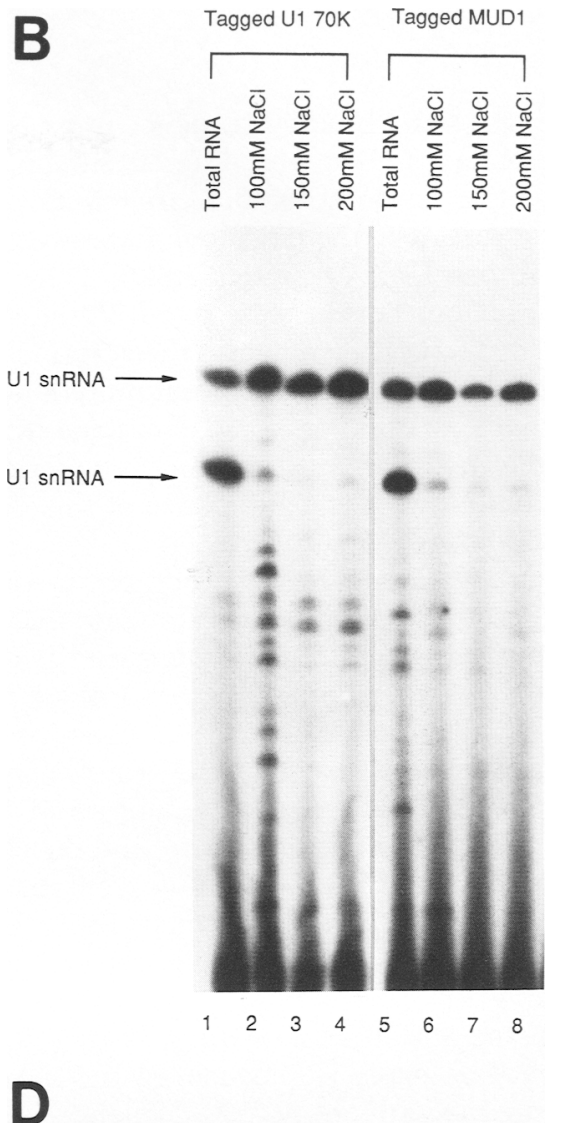

Proximal

Distal

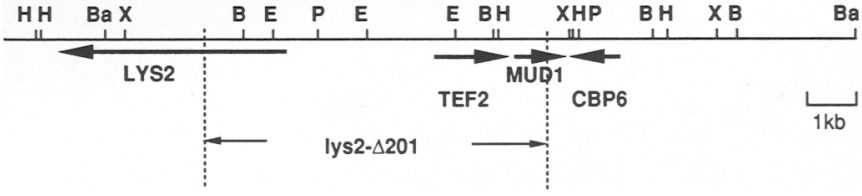

Figure 4. Sequence analyses of the $M U D 1$ gene. $(A)$ Sequence of the MUD1 gene. The MUD1 gene contains a functional intron with conserved splicing signals (indicated by lowercase characters). The nucleotides that are mutated in four mud1 alleles are underlined; the mutant nucleotides are shown above the line of sequence. The mutations of mud1-1 and mud1-2 are identical and create a stop codon at amino acid 84 (indicated as mud1-1). The mutation of mud1-3 changes the initiation codon to an isoleucine, and that of mud1-4 changes amino acid 33 from a tyrosine to an asparagine. The bracket above nucleotides 993-1000 defines the distal breakpoint on chromosome II of the lys2-4201 strains. The breakpoint cannot be defined precisely because of a direct repeat of these $7 \mathrm{bp}$ in the LYS2 gene where they mark the proximal breakpoint of lys2$\Delta 201$. The broken line extending to the left of the bracket indicates that the $5^{\prime}$ half of the MUD1 gene is deleted in the lys2- $\triangle 201$ strains. The numbering of MUD1 starts from the $5^{\prime}$ HindIII site. $(B)$ The MUD1 gene product interacts specifically with U1 snRNA. An extract containing an HA-tagged MUD1 gene product and an extract containing an HA-tagged $\mathrm{U} 170 \mathrm{~K}$ gene were immunoprecipitated with the anti-HA monoclonal antibody 12CA5 as described in Materials and methods. (Lanes 1-4) Tagged U1 70K protein; (lanes 5-8) tagged Mud1 protein; (lanes 1,5) total RNA extracted from the extracts; (lanes 2,6) immunoprecipitation done at 100 mM salt; (lanes 3,7) $150 \mathrm{~mm}$ salt; (lanes 4,8) $200 \mathrm{~mm}$ salt. (C) The amino acid sequence comparison of Mudl with the human UlA protein. The substructure of both RBDs is indicated as described previously (Nagai et al. 1990; Hoffman et al. 1991). Three groups of amino acids that are identical to human U1A but different from U2B" are highlighted with bold characters. $(D)$ The MUD1 gene is located in chromosome II, adjacent to the LYS2, TEF2, and CBP6 genes. The arrows indicate the orientations of the genes. The region deleted in the lys2- 4201 strains is marked by vertical broken lines at the deletion boundaries. Abbreviations for restriction enzymes are $(\mathrm{H})$ HindIII; (Ba) BamHI; (X) XhoI; (B) BglII; (E) EcoRI; (P) PstI. 
RNA molecule, the yeast binding sequence must be different from the metazoan B loop. A different binding site is likely to account for some of the differences in sequence and structure (e.g., the large loop II) between the yeast and the human amino-terminal RBDs.

Although the regions between the two RBDs are very similar in length in both organisms, their amino acid contents appear quite different. The yeast protein is rich in positively charged amino acids, whereas the human protein is proline rich in this region. As the function of this domain is unknown, the significance of this difference is impossible to evaluate.

The high degree of conservation of the carboxy-terminal RBD is impressive and may reflect the fact that this is the most important region of the protein and that the RNA sequence to which it binds-as yet unknown-is similar in yeast and humans. This RBD may interact with a universally conserved region of U1 RNA, another snRNA, or pre-mRNA. Attempts to define its in vitro binding sequence have been unsuccessful, and the entire human U1A protein shows RNA-binding properties indistinguishable from its amino-terminal RBD (LutzFreyermuth et al. 1990; Scherly et al. 1990; Tsai et al. 1991).

To verify that the MUD1 gene is the wild-type equivalent of the mud1-1 locus, we sequenced the mud1-1 gene and compared it with the MUD1 sequence. A single-nucleotide difference was identified, creating a stop codon at amino acid 84 (Fig. 4A). As this mutation should lead to the synthesis of little or no full-length U1A protein (and given that the mud1-1 strain grows normally in combination with a wild-type Ul gene), we considered the possibility that the gene is inessential. This possibility was supported by an analysis of several mutant strains missing chromosomal material near the yeast UlA-coding region (Schirmaier and Philippsen 1984): The sequence of the 1.5-kb U1A-containing subclone identified the yeast translation elongation factor $1 \alpha$-B gene (TEF2) at one end and at the other the yeast nuclear gene CBP6 required for mitochondrial cytochrome $b$ expression. This information mapped MUD1 and CBP6 to chromosome II, next to LYS2 and TEF2 (Fig. 4D). A previously reported deletion of $L Y S 2$ (lys2-4201) was shown to remove $\sim 7 \mathrm{~kb}$ of DNA, including the TEF2 gene, that lies between LYS2 and MUD1. To map the distal breakpoint precisely, we cloned the relevant DNA from several lys2-4201 strains and identified the breakpoint by DNA sequencing. The data showed that the deletion removed all of the MUD1 5'flanking DNA as well as the amino-terminal half of the coding region (Fig. 4A,D). In rich media, these strains grow normally (Schirmaier and Philippsen 1984), as does a deletion of the entire MUD1-coding region (data not shown). These results support the notion that the MUD1 gene is inessential in the presence of a wild-type Ul gene and suggest that the mud1-1 stop codon eliminates much and perhaps all Mud1 activity.

Consistent with the lack of an obvious growth defect of the mud1 mutant strains containing a wild-type U1 gene, the splicing of several introns (e.g., rp51A, actin, and U3) was not detectably affected by the mud1-1 mutation in this background (data not shown). However, by adopting a more sensitive assay for in vivo splicing efficiency, we could demonstrate that Ul snRNP function was nonetheless compromised. We introduced constructs encoding intron-containing $\beta$-galactosidase genes in which enzyme activity requires precise intron removal. Two different intron-containing reporter constructs were compared: One contained an efficiently spliced intron (HZ18; Pikielny and Rosbash 1985), and the other an inefficiently spliced artificial intron (Acc; Legrain and Rosbash 1989). Although the mud1-1 mutation had no detectable effect on the splicing of the efficient HZ18 intron /consistent with the lack of an effect on the splicing of several endogenous intron-containing transcripts; data not shown), it did reduce the splicing efficiency of the inefficient Acc intron by almost 10-fold (Table 1). Presumably, the artificial intron present in the Acc construct is able to detect a deficit in splicing activity that does not impact on efficiently spliced primary transcripts required for vegetative growth. We conclude that the absence of the U1A protein has an adverse effect on Ul snRNP function, even in concert with wild-type U1 RNA, but that this activity is not rate-limiting for the splicing of most normal pre-mRNA substrates.

To test for an effect of the absence of the U1A protein on in vitro splicing, extracts were made from the mud1-1 strains that contained wild-type U1 RNA. With standard pre-mRNA substrates, splicing was indistinguishable from that of the control MUD1 extracts, indicating that the UlA protein is not required for in vitro splicing (data not shown). Although U1 snRNP complex formation was also robust, examination of splicing complexes indicated that there was an assembly phenotype. The early U1 snRNP-containing complexes from a mud1-1 background migrated with a notably faster mobility than those derived from a wild-type background (Fig. 5, lane 2). Identical results were obtained with extracts derived from MUD1 deletion strains (data not shown). These faster-migrating commitment complexes maintained specificity for different mutant substrates, that is, the effects of $5^{\prime}$ splice site and branch point mutations were indistinguishable from their effects on complexes derived from wild-type extracts (data not shown). The mobility difference is rescued by a Mudl-encoding plasmid, as an extract from this strain (mud1-1 + MUD1) was indistinguishable from wild-type (Fig. 5, lane 1). A comparable mobility difference was apparent in U1 snRNP

Table 1. Assay of reporter gene splicing in mudl or MUD1 strains that carry a wild-type U1 snRNA gene

\begin{tabular}{lccc}
\hline Construct & SD5 $(\%)$ & Acc/SD5 $(\%)$ & HZ18/SD5 $(\%)$ \\
\hline Wild type & 100 & 13.0 & 41 \\
mud1-1 & 100 & 1.4 & 41 \\
\hline
\end{tabular}

The absolute values of $\beta$-galactosidase for SD5 (non-intron-containing parent plasmid) were identical for both strains (1700 units) (Legrain and Rosbash 1989) and were defined as $100 \%$. 


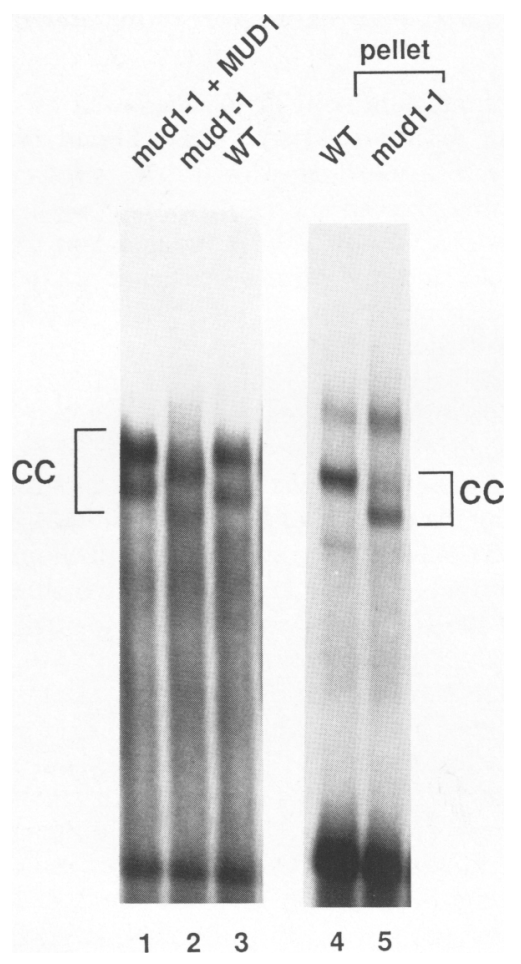

Figure 5. Assembly phenotype of mud1 strain. Whole-cell extracts and a pellet fraction from the strain that carries the mud1-1 mutation in a wild-type U1 background were assayed for commitment complex formation after U2 killing as in Fig. $2 \mathrm{~A}$ (lanes 2,3). The extract and pellet derived from the wild-type strain were assayed in parallel (lanes 3,4 ), as was the extract derived from the mud1-1 strain transformed with the cloned mud1 gene (lane 1). The position of the commitment complexes $(\mathrm{CC})$ is marked with a bracket.

complexes derived from a $0.5 \mathrm{M} \mathrm{KCl}$ pellet fraction, which contains active U1 snRNPs but no loosely associated proteins (Fig. 5, lane 5). As a direct assay of U1 snRNPs (by blotting snRNP gels with a Ul snRNA probe) gave rise to a similar mobility difference (data not shown|, all of these observations are most easily accounted for by the absence of a core U1 snRNP protein in the mud1-1 extracts.

\section{Discussion}

The first gene identified in this synthetic lethal search was the yeast U1A protein. Surprisingly, the gene is inessential in a wild-type Ul RNA background, in vitro as well as in vivo. This finding validates the synthetic lethal strategy for identifying Ul snRNP (or Ul snRNPinteracting/ proteins, as it is very unlikely that this gene would have been identified with a more traditional mutant screen in a wild-type background.

Taken together, our observations suggest either that yeast UIA is not essential for splicing or that another as yet unidentified gene provides a redundant function. We view the latter possibility as less likely based on the following reasons. First, we have been unable to detect the presence of another related gene by low stringency Southern hybridization to the cloned U1A gene (data not shown). Second, the mud1-1 strain (with wild-type U1 snRNA) had a splicing defect as assayed with the inefficiently spliced Acc reporter gene construct (Table 1); the presence of a truly redundant function would provide for wild-type splicing activity in a U1A-deletion strain. Third, the U1 snRNPs from the mud1-1 strain display a uniform mobility shift (Fig. 5) that is easily explained by the absence of one or more snRNP proteins. Identical data were obtained from all of the other deletion strains. We cannot, however, rule out the possibility that there is a loosely associated protein that substitutes for UlA. In any case, the observation that a well-characterized and conserved snRNP protein is inessential suggests that many factors important for splicing may prove to be inessential as defined genetically or biochemically.

Paradoxically, the issue of functional redundancy may be relevant to the synthetic lethal interaction that originally defined the yeast U1A gene. On this basis, it is possible that the U1A protein and the U1 snRNA gene provide somewhat overlapping functions. For example, regions of the U1 RNA and the U1A protein may both function to help fold or maintain the U1 snRNA molecule in an active conformation.

The original U1 snRNA mutant used to identify mud1-1 contained a yeast core deletion as well as an A loop point mutation (Fig. 1A). The lethal interaction with mud1-1 did not require both U1 snRNA mutations and was seen with the yeast core deletion alone, that is, mud1-1 was lethal in combination with a Ul snRNA that only carried the yeast core deletion and was viable in combination with a U1 snRNA that only carried the A loop point mutation (data not shown). On this basis, we propose that a major function of the "extra" information in the 568-nucleotide yeast U1 RNA is to help fold or maintain the universally conserved elements of the molecule in an active configuration. Although structural information will be required to support this suggestion, the reasoning suggests that the large yeast UI RNA molecule might be somewhat less dependent on protein factors than its smaller vertebrate counterpart. A consistent, but not compelling, argument comes from experiments in Xenopus showing that the UI snRNA B loop sequence (i.e., the binding site for the U1A protein) is critical for U1 snRNP function (Hamm et al. 1990). In any case, the inessential nature of the yeast U1A protein and its synthetic lethal interaction with the yeast core deletion suggest that a function of the UIA protein is to help the U1 snRNA achieve an active configuration.

The proposed function implies that snRNPs will follow principles established from some better understood systems of RNA-protein interactions such as ribosomes and group I introns. The data are now quite compelling that one function of ribosomal proteins is to aid the formation of a particular structure in a region of rRNA (Noller et al. 1992). Moreover, several Escherichia coli and yeast ribosomal proteins are inessential; in their absence, cell growth is normal with only subtle phenotypic 
effects (Dabbs 1986; Woolford and Warner 1991). In the case of different group I introns, there is a common core structure but often different peripheral structural elements. The role of at least some of these peripheral elements is to help fold the catalytic core into an active configuration (Celander and Cech 1991; Jaeger et al. 1991; van der Horst et al. 1991; Michel et al. 1992). Proteins also assist; for example, the Neurospora CYT-18 protein helps to stabilize the core in a conformation required for catalytic activity (Guo and Lambowitz 1992; Mohr et al. 1992). In this view, one can imagine that both the UIA protein and the "extra" RNA of yeast U1 snRNA perform analogous functions to stabilize the conserved regions of Ul snRNA in an optimal configuration.

\section{Materials and methods}

Strains used in mutagenesis

The mud1-4 allele was obtained from an early pilot experiment after UV mutagenesis of the strain XLY140 (MAT $\alpha$, trp1-289, ura3-52, leu2-3, leu2-112, his3- $\Delta 1$, cyh $^{\mathrm{R}}$, snr19::LEU2, pXL46, pXL18-84), which differs from XLY216 by the configuration of the genes at the snr19 locus. The strains used in the mutagenesis were XLY219 [MATa, trp1-289, ura3-52, leu2-3, 112, arg4 $\left(\mathrm{RV}^{-}\right)$, ade2, snr19A::ADE2, pXL46, pXL18-84] and XLY216 (MATa, trp1-289, ura3-52, leu2-3, 112, his3- $\Delta 1, c y h^{\mathrm{R}}$, snr194::ADE2, pXL46, pXL18-84), where pXL46 is the URA3GAL-U1 plasmid.

\section{Mutagenesis and genetic screen}

The starting strains were grown in $\operatorname{Trp}^{-} \mathrm{Ura}^{-}+3 \%$ galactose $+1 \%$ sucrose medium before mutagenesis. UV mutagenesis was carried out with a UV lamp (UV Stratalinker 1800, Stratagene) on individual cells plated on the $\operatorname{Trp}^{-} \mathrm{Ura}^{-}+$galactose plates. Ethylmethane sulfonate (EMS) mutagenesis was carried out in cell suspension according to standard protocols (Lawrence 1991), and mutagenized cells were plated immediately on the plates described above. The viable colonies $15-15 \%$ of the mutagenized cells) on the master plates were replicaplated to 5-FOA plates and grown at $30^{\circ} \mathrm{C}$. The master plates and replica plates were compared, and the missing colonies on the replica plates were identified as having candidate mutations that synergize with the mutant Ul snRNA to cause a lethal phenotype. The corresponding mutant colonies /called mud mutants) were collected from the master plates and examined for their auxotrophic markers and their inability to grow in medium containing glucose. The mud1-1 and mud1-2 alleles were obtained after UV mutagenesis of the strain XLY219, whereas the mud1-3 allele was recovered from EMS mutagenesis of the same strain.

\section{In vitro spliceosome and commitment complex assembly}

The strains were grown in medium containing $4 \%$ glucose for $16 \mathrm{hr}$ before RNA or extracts were prepared. The extracts were prepared using a modified miniextract protocol (Séraphin and Rosbash 1989; Abovich et al. 1990). In vitro spliceosome assembly was performed according to standard protocols (Séraphin and Rosbash 1991), and the U2 killing of extracts and pellet fractions was as described (Liao et al. 1992). For gel assays, each figure comes from a single gel and autoradiographic exposure. The primer used for reverse transcription of U1 snRNA is DT13, which is complementary to nucleotides 535-551 of U1 snRNA.
Cloning of the wild-type MUDl gene and mapping of mudl mutants

The original mud1-1 mutant (Ym8) was used as the host for transformation with a wild-type yeast library based on the LEU2-CEN vector $\mathrm{p} 366$. Transformants were selected on Leu ${ }^{-}$ plates containing glucose and confirmed by replica-plating to 5-FOA. Total RNA was made from independent transformants after 5-FOA selection, and primer extension of U1 snRNA was employed to distinguish the strains that were rescued by the MUD1 clones, which would express only mutant U1 snRNA, from those rescued by the wild-type U1 snRNA gene, which would express both the full-length U1 snRNA and the mutant U1 snRNA. The MUD1 clones were recovered from yeast by transforming E. coli. To prove the existence of a functional intron and to map the precise splice sites, total RNA from a wildtype strain DBY745- $\triangle \mathrm{A}$ was reverse transcribed and PCR amplified with primers DT2325 and DT2311, which are identical to nucleotides 425-444 and complementary to nucleotides 706723 , respectively (Fig. 4A). For the sequencing of the four mud1 alleles, yeast DNA from these mud1 mutant strains was amplified and sequenced by PCR using primers DT2312 and DT2313, which are identical to the first 18 nucleotides and complementary to the last 18 nucleotides of the $1.5-\mathrm{kb}$ HindIII fragment (see Fig. 4A), respectively. The lys2-4201 and the control LYS2 strains were a kind gift from Cora Ann Styles in G. Fink's laboratory (Whitehead Institute, MIT, Cambridge, MA). These strains are L983 (LYS2), L1535 (lys2-4201), L3282(lys2-4201), and L4576 (lys2-4201). The deletion junction of lys2-4201 was cloned from the three lys2- $\Delta 201$ strains by PCR using primer DT2428 for the LYS2 gene (5' -CCAGCAGGCATGACATCT- $3^{\prime}$ ) and DT2323, which is complementary to nucleotides 11901213 of MUD1. DT2323 was also used for sequencing the deletion junction.

\section{Fractionation of extracts}

Extracts were fractionated by centrifugation, as in Abovich et al. (1990), except (1) the $\mathrm{KCl}$ was at either $50 \mathrm{~mm}$ (the normal concentration in the extract, called low salt) or $500 \mathrm{~mm}$ (called high salt); (2) the centrifugation was for $4 \mathrm{hr}_{\text {; }}$ and (3) buffer D contained $0.05 \%$ NP- 40 for resuspending the pellets.

\section{Immunoprecipitation}

Strains were constructed containing HA-tagged versions of the U1 70K gene and the MUD1 gene essentially as described previously for the PRP6 gene (Abovich et al. 1990). In both cases, the epitope was placed at the carobxyl terminus of the protein, and the tagged gene was used to complement a gene knockout. Extracts were made from these two strains, and the 12CA5 antibody was used to immunoprecipitate the tagged proteins from the extracts. RNA was extracted from the precipitates and subjected to primer extension using the primer DT58 for U2 snRNA and DT586 for U1 snRNA (Liao et al. 1990). Extract preparation, immunoprecipitation, RNA extraction, and primer extension were as described (Abovich et al. 1990).

\section{Acknowledgments}

We thank B. Séraphin, H. Colot, C. Pikielny, and P. Zamore for comments on the manuscript. H. Colot, D. Kenan, J. Keene, and I. Mattaj provided insight into the sequence comparisons. We thank L. Luo for helpful discussions, encouragement, and for making the yeast genomic library DNA preparations used in cloning the MUD1 gene. The yeast genomic library was a kind 
gift of P. Hieter. The $A D E 2$ gene, as well as the 1ys2- 4201 strains, were obtained from C. A. Styles and G. Fink. This work was supported by a grant from the National Institutes of Health (GM23549) to M.R.

The publication costs of this article were defrayed in part by payment of page charges. This article must therefore be hereby marked "advertisement" in accordance with 18 USC section 1734 solely to indicate this fact.

\section{References}

Abovich, N., P. Legrain, and M. Rosbash. 1990. The yeast PRP6 gene encodes a U4/U6 small nuclear ribonucleoprotein particle (snRNP) protein, and the PRP9 gene encodes a protein required for U2 snRNP binding. Mol. Cell. Biol. 10: 64176425.

Barabino, S., B.J. Blencowe, U. Ryder, B.S. Sproat, and A.I. Lamond. 1990. Targeted snRNP depletion reveals an additional role for mammalian Ul snRNP in spliceosome assembly. Cell 63: 293-302.

Bartkiewicz, M., H. Gold, and S. Altman. 1989. Identification and characterization of an RNA molecule that copurifies with RNase P activity from HeLa cells. Genes \& Dev. 3: 488-499.

Boeke, J.D., F. Lacroute, and G.R. Fink. 1984. A positive selection for mutants lacking orotidine- 5 '-phosphate decarboxylase activity in yeast: 5-Fluoro-orotic acid resistance. Mol. Gen. Genet. 197: 345-346.

Celander, D.W. and T.R. Cech. 1991. Visualizing the higher order folding of a catalytic RNA molecule. Science 251: 401407.

Dabbs, E.R. Mutant studies on the prokaryotic ribosome. 1986. In Structure, function, and genetics of ribosomes (ed. B. Hardesty and G. Kramer), pp. 733-748. Springer-Verlag, New York.

Donahue, T.F. and A.M. Cigan. 1990. Sequence and structure requirements for efficient translation in yeast. Methods Enzymol. 185: 366-372.

Frank, D., B. Patterson, and C. Guthrie. 1992. Synthetic lethal mutations suggest interactions between U5 small nuclear RNA and four proteins required for the second step of splicing. Mol. Cell. Biol. 12: 5197-5205.

Green, M.R. 1991. Biochemical mechanisms of constitutive and regulated pre-mRNA splicing. Annu. Rev. Cell Biol. 7: 559599.

Guo, Q. and A.M. Lambowitz. 1992. A tyrosyl-tRNA synthetase binds specifically to the group I intron catalytic core. Genes \& Dev. 6: 1357-1372.

Guthrie, C. 1991. Messenger RNA splicing in yeast: Clues to why the spliceosome is a ribonucleoprotein. Science 253: 157-163.

Hamm, J., N.A. Dathan, D. Scherly, and I.W. Mattaj. 1990. Multiple domains of U1 snRNA, including U1 specific protein binding sites, are required for splicing. EMBO I. 9: 12371244.

Hoffman, D.W., C.C. Query, B.L. Golden, S.W. White, and J.D. Keene. 1991. RNA-binding domain of the A protein component of the U1 small nuclear ribonucleoprotein analyzed by NMR spectroscopy is structurally similar to ribosomal proteins. Proc. Natl. Acad. Sci. 88: 2495-2499.

Huffaker, T.C., M.A. Hoyt, and D. Botstein. 1987. Genetic analysis of the yeast cytoskeleton. Annu. Rev. Genet. 21: 259284.

Jamison, S.F., A. Crow, and M.A. Garc a-Blanco. 1992. The spliceosome assembly pathway in mammalian extracts. Mol.
Cell. Biol. 12: 4279-4287.

Jaeger, L., E. Westhof, and F. Michel. 1991. Function of P11, a tertiary base-pairing in self-splicing introns of subgroup IA. $J$. Mol. Biol. 221: 1153-1164.

Jessen, T-H., C. Oubridge, C.H. Teo, C. Prichard, and K. Nagai. 1991. Identification of molecular contacts between the Ul A small nuclear ribonucleoprotein and U1 RNA. EMBO $J$. 10: 3447-3456.

Kranz, J.E. and C. Holm. 1990. Cloning by function: An alternative approach for identifying yeast homologs of genes from other organisms. Proc. Natl. Acad. Sci. 87: 6629-6633.

Kretzner, L., A. Krol, and M. Rosbash. 1990. Saccharomyces cerevisiae U1 small nuclear RNA secondary structure contains both universal and yeast-specific domains. Proc. Natl. Acad. Sci. 87: 851-855.

Lamm, G.M., B.J. Blencowe, B.S. Sproat, A.M. Iribarren, U. Ryder, and A.I. Lamond. 1991. Antisense probes containing 2-aminoadenosine allow efficient depletion of U5 snRNP from HeLa splicing extracts. Nucleic Acids Res. 190: 31933198.

Lawrence, C.W. 1991. Classical mutagenesis techniques. Methods Enzymol. 194: 273-281.

Legrain, P. and M. Rosbash. 1989. Some cis- and trans-acting mutants for splicing target pre-mRNA to the cytoplasm. Cell 57: 573-583.

Legrain, P., B. Séraphin, and M. Rosbash. 1988. Early commitment of yeast pre-mRNA to the spliceosome pathway. Mol. Cell. Biol. 8: 3755-3760.

Legrain, P., C. Chapon, E. Schwob, R. Martin, M. Rosbash, and B. Dujon. 1991. Cloning of the two essential yeast genes, $P R P 6$ and $P R P 9$, and their rapid mapping, disruption and partial sequencing using a linker insertion strategy. Mol. \& Gen. Genet. 225: 199-202.

Liao, X., L. Kretzner, B. Séraphin, and M. Rosbash. 1990. Universally conserved and yeast-specific Ul snRNA sequences are important but not essential for U1 snRNP function. Genes \& Dev. 4: 1766-1774.

Liao, X.C., H.V. Colot, Y. Wang, and M. Rosbash. 1992. Requirements for U2 snRNP addition to yeast pre-mRNA. Nucleic Acids Res. 20: 4237-4245.

Lutz-Freyermuth, C., C.C. Query, and J.D. Keene. 1990. Quantitative determination that one of two potential RNA-binding domains of the A protein component of the U1 small nuclear ribonucleoprotein complex binds with high affinity to stem-loop II of U1 RNA. Proc. Natl. Acad. Sci. 87: 63936397.

Maniatis, T. and R. Reed. 1987. The role of small nuclear ribonucleoprotein particles in pre-mRNA splicing. Nature 325: 673-678.

Michaud, S. and R. Reed. 1991. An ATP-independent complex commits pre-mRNA to the mammalian spliceosome assembly pathway. Genes \& Dev. 5: 2534-2546.

Michel, F., L. Jaeger, E. Westhof, R. Kuras, F. Tihy, M.-Q. Xu, and D.A. Shub. 1992. Activation of the catalytic core of a group I intron by a remote $3^{\prime}$ splice junction. Genes \& Dev. 6: 1373-1385.

Mohr, G., A. Zhang, J.A. Gianelos, M. Belfort, and A.M. Lambowitz. 1992. The neurospora CYT-18 protein suppresses defects in the phage T4 $t d$ intron by stabilizing the catalytically active structure of the intron core. Cell 69: 483-494.

Myslinski, E., V. Segault, and C. Branlant. 1990. An intron in the genes for the U3 small nucleolar RNAs of the yeast Saccharomyces cerevisiae. Science 247: 1213-1216.

Nagai, K., C. Oubridge, T.H. Jessen, J. Li, and P.R. Evans. 1990. Crystal structure of the RNA-binding domain of the U1 small nuclear ribonucleoprotein A. Nature 348: 515-520. 
Noller, H.F., V. Hoffarth, and L. Zimniak. 1992. Unusual resistance of peptidyl transferase to protein extraction procedures. Science 256: 1416-1419.

Pikielny, C.W. and M. Rosbash. 1985. mRNA splicing efficiency in yeast and the contribution of nonconserved sequences. Cell 41: 119-126.

Rosbash, M. and B. Séraphin. 1991. Who's on first? The Ul snRNP-5' splice site interaction and splicing. Trends Biochem. Sci. 16: 187-190.

Ruby, S.W. and J. Abelson. 1988. An early hierarchic role of U1 small nuclear ribonucleoprotein in spliceosome assembly. Science 242: 1028-1035. 85.

Scherly, D., W. Boelens, N.A. Dathan, W.J. Van Venrooij, and I.W. Mattaj. 1990. Major determinants of the specificity of interaction between small nuclear ribonucleoproteins U1A and U2B" and their cognate RNAs. Nature 345: 502-506.

Scherly, D., C. Kambach, W. Boelens, W.J. Van Venrooij, and I.W. Mattaj. 1991. Conserved amino acid residues within and outside of the amino-terminal ribonucleoprotein motif of U1A small nuclear ribonucleoprotein involved in U1 RNA-binding. I. Mol. Biol. 219: 577-584.

Schirmaier, F. and P. Philippsen. 1984. Identification of two genes coding for the translation elongation factor EF- $1 \alpha$ of $S$. cerevisiae. EMBO I. 3: 3311-3315.

Séraphin, B. and M. Rosbash. 1989. Identification of functional U1 snRNA-pre-mRNA complexes committed to spliceosome assembly and splicing. Cell 59: 349-358.

- 1991. The yeast branch point sequence is not required for the formation of a stable U1 snRNP-pre-mRNA complex and is recognized in the absence of U2 snRNA. EMBO $/$. 10: $1209-1216$.

Séraphin, B., N. Abovich, and M. Rosbash. 1991. Genetic depletion indicates a late role for U5 snRNP during in vitro spliceosome assembly. Nucleic Acids Res. 19: 3857-3860.

Simon, M.A., D.D.L. Bowtell, G.S. Dodson, T.R. Laverty, and G.M. Rubin. 1991. Rasl and a putative guanine nucleotide exchange factor perform crucial steps in signaling by the sevenless protein tyrosine kinase. Cell 67: 701-716.

Smith, V. and B.G. Barrell. 1991. Cloning of a yeast Ul snRNP 70K protein homolog: Functional conservation of an RNAbinding domain between humans and yeast. EMBO $/$. 10: $2627-2634$.

Steitz, J.A., D.L. Black, V. Gerke, K.A. Parker, A." Kramer, D. Frendewey, and W. Keller. 1988. Functions of the abundant U-snRNPs. In Structure and function of major and minor small nuclear ribonucleoprotein particles (ed. M.L. Birnstiel), pp. 115-154. Springer-Verlag, Berlin.

Tsai, D.E., D.S. Harper, and J.D. Keene. 1991. U1-snRNP-A protein selects a ten nucleotide consensus sequence from a degenerate RNA pool presented in various structural contexts. Nucleic Acids Res. 19: 4931-4936.

van der Horst, G., A. Christian, and T. Inoue. 1991. Reconstitution of a group I intron self-splicing reaction with an activator RNA. Proc. Natl. Acad. Sci. 88: 184-188.

Vijayraghavan, U., M. Company, and J. Abelson. 1989. Isolation and characterisation of pre-mRNA splicing mutants in Saccharomyces cerevisiae. Genes \& Dev. 3: 1206-1216.

Wilson, I.A., H.L. Niman, R.A. Houghten, M. Cherenson, L. Connolly, and R.A. Lerner. 1984. The structure of an antigenic determinant in a protein. Cell 37: 767-778.

Woolford, J.L., Jr. and J.R. Warner. 1991. The ribosome and its synthesis. In The molecular and cellular biology of the yeast Saccharomyces: Genome dynamics, protein synthesis, and energetics (ed. J.R. Broach, J.R. Pringle, and E.W. Jones), pp.
587-626. Cold Spring Harbor Laboratory Press, Cold Spring Harbor, New York.

Zavanelli, M.I. and M. Ares, Jr. 1991. Efficient association of U2 snRNPs with premessenger RNA requires an essential U2 RNA structural element. Genes \& Dev. 5: 2521-2533. 


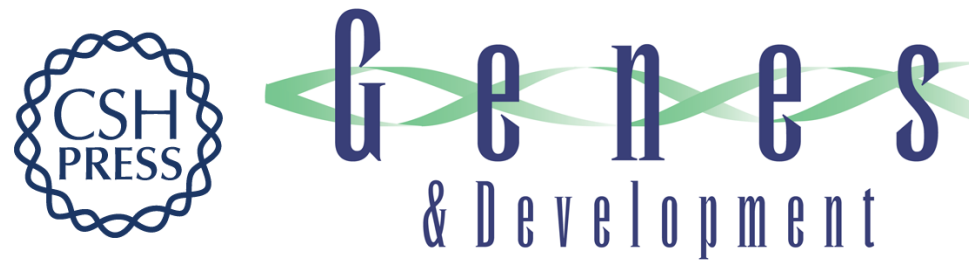

\section{An enhancer screen identifies a gene that encodes the yeast U1 snRNP A protein: implications for snRNP protein function in pre-mRNA splicing.}

X C Liao, J Tang and M Rosbash

Genes Dev. 1993, 7:

Access the most recent version at doi:10.1101/gad.7.3.419

References This article cites 50 articles, 21 of which can be accessed free at: http://genesdev.cshlp.org/content/7/3/419.full.html\#ref-list-1

License

Email Alerting Service

Receive free email alerts when new articles cite this article - sign up in the box at the top right corner of the article or click here.

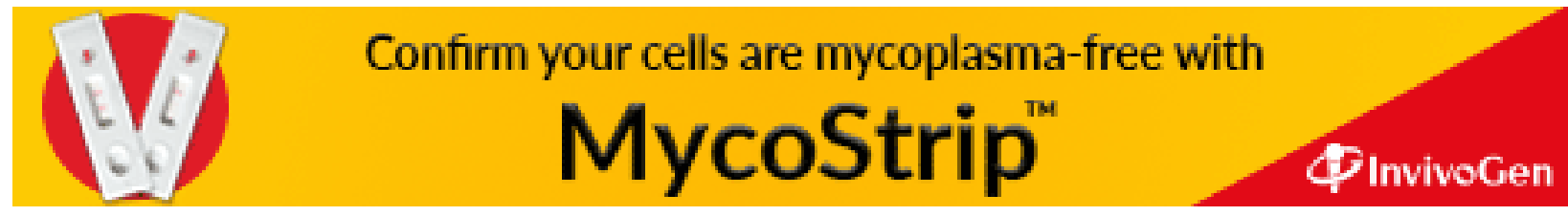

